PROYECTOS INTEGRADORES. UNA ESTRATEGIA PARA DESARROLLAR PROYECTOS SUSTENTABLES EN EL AULA

\title{
PROYECTOS INTEGRADORES. UNA ESTRATEGIA PARA DESARROLLAR PROYECTOS SUSTENTABLES EN EL AULA
}

\section{INTEGRATING PROJECTS. A STRATEGY FOR DEVELOPING SUSTAINABLE PROJECTS IN THE CLASSROOM}

\author{
Mónica Leticia Acosta Miranda*, Celina Hiosilene Ocampo Ponce**, \\ Misael Samuel Corona Calvario***
}

* Maestra en Administración. Instituto Tecnológico de Cuautla.

** Maestra en Administración de Negocios. Instituto Tecnológico de Cuautla. hiosy2010@live.com.mx *** Maestro en Administración de la Pequeña y Mediana Empresa. Instituto Tecnológico de Cuautla. samuel.corona@itcuautla.edu.mx

Dirección para recibir correspondencia: monycaacosta@yahoo.com 
PROYECTOS INTEGRADORES. UNA ESTRATEGIA PARA DESARROLLAR PROYECTOS SUSTENTABLES EN EL AULA

\section{RESUMEN}

OBJETIVO: Emplear los proyectos integradores que se desarrollan en la carrera de Contador Público que se oferta en el Instituto Tecnológico de Cuautla, plantel del Tecnológico Nacional de México (TecNM), como una estrategia curricular integral en la formación y evaluación de competencias, al tiempo que genera proyectos emprendedores que aborden problemas del entorno considerando principalmente el cuidado del medio ambiente.

MATERIAL Y MÉTODO: Se ha desarrollado al seno de la academia, tomando como referencia la normativa vigente emitida por el TecNM, seleccionando las asignaturas y su aportación al proyecto, al mismo tiempo que se realizan actividades coordinadas enfocadas al desarrollo del mismo.

RESULTADOS: Los equipos presentan sus proyectos en diversos foros y al término del $7^{\circ}$. Semestre, en la Presentación de Proyectos del plantel. Algunos se han incorporado a la Incubadora de empresas del instituto, con la finalidad de obtener financiamiento y convertirlos en empresas generadoras de empleo.

CONCLUSIONES: Los proyectos integradores apoyan y vinculan el proceso enseñanzaaprendizaje con problemas reales, que permitan a los estudiantes desarrollar capacidades de trabajo en equipos multidisciplinarios, así como identificar, formular y resolver problemas y tomar conciencia de su responsabilidad profesional y social.

PALABRAS CLAVE: Proyecto integrador. Emprendedores. Nuevas empresas. 
PROYECTOS INTEGRADORES. UNA ESTRATEGIA PARA DESARROLLAR PROYECTOS SUSTENTABLES EN EL AULA

\section{ABSTRACT}

OBJECTIVE: Use the integrating projects that are developed in the career of Public Accountant that is offered in the Technological Institute of Cuautla, campus of the National Technological Institute of Mexico (TecNM), as an integral curricular strategy in the formation and evaluation of competencies, at the same time that it generates entrepreneurial projects that address environmental problems, considering mainly the care of the environment.

MATERIAL AND METHOD: It has been developed within the academy, taking as reference the current regulations issued by the TecNM, selecting the school subjects and their contribution to the project, while coordinated activities are carried out focused on the development of the same objective.

RESULTS: The teams present their projects in various forums and at the end of the 7th. Semester, in the Presentation of Projects from the campus.. Some of them have joined the enterprises Incubator of the institute, in order to obtain financing and convert them into employment generating companies.

CONCLUSIONS: The integrative projects support and link the teaching-learning process with real problems, which allow students to develop work capacities in multidisciplinary teams, as well as identify, formulate and solve problems and become aware of their professional and social responsibility.

KEYWORDS: Integrating project. Entrepreneurs. New companies. 
PROYECTOS INTEGRADORES. UNA ESTRATEGIA PARA DESARROLLAR PROYECTOS SUSTENTABLES EN EL AULA

\section{INTRODUCCIÓN}

Actualmente, las Instituciones de Educación Superior tienen el compromiso social de preparar mejor a los jóvenes para el mundo laboral, desarrollando en ellos competencias que les permitan realizar la transferencia y movilización de conocimientos a situaciones de trabajo (Molina Ruíz, 2007). Frente a las exigentes demandas sociales se perfila la necesidad de desarrollar proyectos de investigación que den solución a problemas del contexto. Vincular el proceso de enseñanza-aprendizaje con problemas reales del entorno, lograr que los estudiantes demuestren la capacidad para trabajar en equipos multidisciplinarios; la capacidad para identificar, formular y resolver problemas de su área; la comprensión de la responsabilidad profesional y ética; la capacidad de comunicarse eficazmente y una educación suficientemente amplia para comprender el impacto de las soluciones en un contexto global, económico, ambiental y social (Paz, 2007).

Los planteles que conforman el TecNM desarrollan sus actividades de docencia basados en el Modelo Educativo para el Siglo XXI. Formación y Desarrollo de Competencias Profesionales (Dirección General de Educación Superior Tecnológica, 2012b), que hace patente la importancia de la investigación en la formación de profesionistas, afirmando que ésta es una forma de generar conocimientos pertinentes y de actualidad y sirve para enriquecer el acervo cultural. La investigación es una estrategia útil para vincular a los Institutos Tecnológicos con el entorno regional, nacional y mundial.

En los Institutos Tecnológicos de México se busca formar ciudadanos de clase mundial que aprendan en la vida y para la vida. Sus egresados se caracterizan por una activa participación ciudadana basada en principios éticos, aptos para contribuir en la construcción de la sociedad del conocimiento, a través del impulso de proyectos de investigación y desarrollo tecnológico, para su propio desarrollo profesional y humano, y con el desarrollo de su comunidad y del país (Dirección General de Educación Superior Tecnológica, 2012b).

Los proyectos integradores constituyen una estrategia curricular que permite generar una nueva manera en que los estudiantes desarrollen diversos tipos de competencias a lo largo de su trayectoria en el instituto, lo que significa que debe de contemplar oportunidades para aprender a actuar de forma integral y no individualizada. Todo proyecto busca abordar 
PROYECTOS INTEGRADORES. UNA ESTRATEGIA PARA DESARROLLAR PROYECTOS SUSTENTABLES EN EL AULA

problemas en el contexto, y en ese sentido es la estrategia más integral para la formación y evaluación de las competencias (Tobón S., 2010b).

El proyecto integrador facilita el aprendizaje del estudiante a través de la realización de un conjunto de actividades, en la resolución desde uno hasta varios problemas de contexto incorporando el saber, el saber ser y el saber hacer de forma integrada en las diversas actividades del proyecto.

El proyecto integrador permite, además la vinculación de las instituciones educativas y la sociedad en su conjunto, donde los conocimientos, destrezas, y habilidades del estudiante van más allá del ámbito escolar y le permiten acumular experiencia a través de la respuesta a una problemática real de su entorno, acorde a la misión y visión del TecNM.

A través de los proyectos integradores es posible la articulación entre la formación de competencias profesionales que se ofrecen en el aula y las necesidades de los sectores productivos y sociales. También es útil para ajustar e innovar los desempeños profesionales con las demandas del sector productivo y realizar una evaluación de su pertinencia y actualidad.

En el Instituto Tecnológico de Cuautla, plantel perteneciente al Sistema Nacional de Institutos Tecnológicos, hoy TecNM, en particular en la carrera de Contador Público, la Academia de Ciencias Económico-Administrativas, siempre ha existido la preocupación por el desarrollo económico, social y ecológico de la zona que se atendido de diversas formas.

Desde hace tres años, al seno de la academia, se comenzó a trabajar con los proyectos integradores, considerando que constituían la mejor forma de desarrollar las competencias planteadas en los programas de estudio de las diversas materias con proyectos que pudieran tener un verdadero impacto en la región y no trabajos realizados solo en el papel y uno diferente para cada asignatura.

De esta forma, se ha logrado la creación de microempresas que no solo generan autoempleo, sino que poco a poco, se constituyen en fuentes de empleo en la localidad. La preocupación ahora es crear proyectos que además tengan un impacto en la ecología y el medio ambiente. 
PROYECTOS INTEGRADORES. UNA ESTRATEGIA PARA DESARROLLAR PROYECTOS SUSTENTABLES EN EL AULA

Bajo esta mecánica surge Econtagy, empresa preocupada por la producción y comercialización de eco-tecnología como un mecanismo para preservar el medio ambiente, cuyo primer producto en el mercado consiste en un protector para teléfono celular que tiene integrado una celda fotovoltaica, esto con el fin de minimizar el consumo de energía eléctrica y aprovechar la luz solar, bastante abundante en la zona.

En este trabajo se presenta la experiencia de ligar los proyectos integradores con el emprendedurismo y el desarrollo sustentable desde el aula.

\section{MARCO TEÓRICO}

\section{El emprendedurismo en México}

En la actualidad, la mayor parte de los países le está apostando a los emprendedores como fuente de su futuro económico ya que estos otorgan beneficios a la sociedad con su desempeño. En los últimos tiempos cada día escuchamos más de esta actividad empresarial y nos damos cuenta de la importancia de esta palabra, que se oye más a menudo y de manera trascendente.

Existen diversos conceptos de emprendedor y de emprendedurismo, algunos definen emprendedurismo como: "actividad profesional de los llamados emprendedores". De igual forma definen a los emprendedores: "Personas extrañas, con inquietudes nunca entendidas, con obsesiones intolerables, e ideas irrealizables, personas que se aferran a una idea y no están satisfechos hasta realizarla; para ellos lo imposible no existe, a pesar de que su idea sea para la mayoría descabellada, reúnen los recursos y herramientas para llevarla a cabo y transformarla a una idea considerada innovadora, personas inquietas con ganas de hacer, con mentes que sólo ellos entienden "locos de los negocios", personas que buscan retos, probar y probarse. Buscan metas cada vez más altas.

Ser emprendedor es querer cambiar el mundo a partir de ideas, es tener la fuerza y capacidad de construir un imperio a partir de cenizas y reconstruirlo cada vez que sea necesario. E emprendimiento es algo que está presente en nuestro país, se encuentra dentro de nuestra sangre y debe de salir a la luz. 
PROYECTOS INTEGRADORES. UNA ESTRATEGIA PARA DESARROLLAR PROYECTOS SUSTENTABLES EN EL AULA

Se denomina emprendedor a aquella persona que sabe descubrir, identificar una oportunidad de negocios en concreto y entonces se dispondrá a organizar o conseguir los recursos necesarios para comenzarla y llevarla al éxito. Generalmente, este término, se aplica para designar a las personas que de la nada, solo con la idea, logran crear una empresa.

Las principales características de un emprendedor son: la flexibilidad, el dinamismo, la creatividad, orientación hacia la aventura y el riesgo que forman parte del perfil de la persona emprendedora.

En México, siete de cada 10 empleos se genera en una PyME y, de acuerdo al Índice de Espíritu Empendedor de Amway ${ }^{1}$ en su reporte global de emprendedores 2015, México es el segundo país con mayor factibilidad para el emprendedurismo.

En la encuesta realizada en 44 países para dicho reporte el 76\% de los mexicanos respondió que considera contar con las habilidades y los recursos necesarios para iniciar un negocio, solo debajo del $81 \%$ de los chinos.

Dicho reporte encontró también que nuestro país tiene la segunda actitud más positiva ante el emprendedurismo y el $84 \%$ de los encuestados menores de 35 años se imagina a si mismo emprendiendo un negocio, destacando el espíritu emprendedor que sobresale en las mujeres

Desde siempre México ha sido un país compuesto por una gran cantidad de emprendedores ya sea por naturaleza o por necesidad. Con negocios propios, pequeñas tiendas de abarrotes, tianguis, puestos de tacos, donde existe una increíble habilidad para superar los límites impuestos por la piratería y proyectando nuevas fronteras de la autenticidad y creatividad, estos son algunos ejemplos de cómo los mexicanos han desarrollado ese instinto emprendedor mal orientado, donde a cualquier problema se le encuentra solución con una destreza que cualquier corporativo quisiera tener. El problema es cómo orientar este empuje hacia escenarios más positivos y enriquecedores para la comunidad.

La educación es un factor muy importante para llevar esta habilidad por el camino adecuado, muchas personas emprendedoras, no saben que lo son, no saben qué es esto y esas inquietudes que sienten no saben en que enfocarlas. El apoyo económico a emprendedores en México es grandísimo y es un deber aprovecharlo - Las incubadoras empresariales y las

${ }^{1}$ American Way, compañía de marketing multinivel. 
PROYECTOS INTEGRADORES. UNA ESTRATEGIA PARA DESARROLLAR PROYECTOS SUSTENTABLES EN EL AULA

creadas al interior de las Instituciones de Educación Superior ayudan a llevar esto a cabo, hay que entender que el futuro de México depende de los pequeños negocios, de dar empleo a la mayor cantidad de personas posible, no es posible esperar a que las empresas den trabajo a todos, urge crear trabajos para asegurar un futuro económico prometedor para nuestro país.

\section{Los proyectos integradores en el Tecnológico Nacional de México}

Los proyectos integradores son una excelente estrategia curricular que permite el desarrollo y formación de competencias en la solución de problemas de contexto en la educación superior tecnológica. Relacionados con el tema encontramos algunos conceptos fundamentales que explicaremos brevemente.

\section{Competencia}

El TecNM opera con el Modelo Educativo para el siglo XXI. Formación y Desarrollo de Competencias Profesionales por lo que hablar del concepto de competencias profesionales es fundamental.

Las competencias son actuaciones integrales para identificar, interpretar, argumentar y resolver problemas con idoneidad y compromiso ético, movilizando los diferentes saberes: ser, hacer y conocer (Tobón S., 2010a).

Las competencias se redactan a través de un desempeño (uno o varios verbos en presente o infinitivo), el objeto conceptual (objeto sobre el cual recae la acción), una finalidad (es lo que responde a la pregunta ¿para qué de la competencia?) y finalmente una condición de referencia (es el contexto de la competencia, el cual permite valorar su calidad).

Para el TecNM una competencia es la integración y aplicación estratégica de conocimientos, procedimientos y actitudes necesarios para la solución de problemas de contexto, con una actuación profesional, ética, eficiente y pertinente en escenarios laborales heterogéneos y cambiantes (TecNM, 2012).

Una forma de aplicar estos conocimientos, procedimientos y actitudes es a través de los proyectos. ¿Qué es un proyecto? ¿Qué implica? 
PROYECTOS INTEGRADORES. UNA ESTRATEGIA PARA DESARROLLAR PROYECTOS SUSTENTABLES EN EL AULA

\section{Proyecto}

Es el conjunto de elementos o partes interrelacionados de una estructura diseñada para lograr los objetivos específicos, o resultados proyectados con base en necesidades detectadas y que han sido diseñados como propuesta para presentar alternativas de solución a problemas planteados en él, por lo que en el proyecto se debe diseñar la estrategia metodológica, a partir de la cual se considera que puede obtener el nuevo conocimiento como solución al problema (Whitney, 1970), (Van Dalen \& Meyer, 1974), (Tamayo y Tamayo M., 1994), (Tamayo y Tamayo M., 1999) у (Tamayo y Tamayo M., 2003).

El Instituto Colombiano para el Fomento de la Educación Superior (ICFES, 1999) considera que: "el término proyecto en este sentido genérico no significa más que el planeamiento de algo. El término proyecto se deriva de los verbos latinos proicere y proiectare, que significan arrojar algo hacia adelante. Entonces proyecto significa el pensamiento o el designio de hacer algo; la disposición que se hace de algo, anotando todos los elementos que deben concurrir para su logro; la planeación y organización previa de todas las tareas y actividades necesarias para alcanzar algo".

De aquí, se puede identificar los elementos básicos de un proyecto:

- El proyecto es el planeamiento de algo.

- En el proyecto se indican y justifican los conjuntos de acciones necesarias para alcanzar un objetivo específico determinado.

- Las acciones se planifican en el proyecto dentro de ciertos parámetros de concepción, de tiempo y de recursos.

Como conclusión, un proyecto podría describirse como el planeamiento de algo, en el cual se indican y justifican los conjuntos de acciones necesarias para alcanzar un objetivo determinado. Dentro de determinados parámetros de concepción, tiempo y recursos.

\section{Proyecto integrador}

Un proyecto integrador es una estrategia didáctica que consiste en realizar un conjunto de actividades articuladas entre sí, con un inicio, un desarrollo y un final con el propósito de identificar, interpretar, argumentar y resolver un problema del contexto, y así contribuir a formar 
PROYECTOS INTEGRADORES. UNA ESTRATEGIA PARA DESARROLLAR PROYECTOS SUSTENTABLES EN EL AULA

una o varias competencias del perfil de egreso, teniendo en cuenta el abordaje de un problema significativo del contexto disciplinar-investigativo, social, laboral-profesional, (López Rodríguez, 2012).

El proyecto integrador es una estrategia metodológica y evaluativa de investigación, direccionada al planteamiento y solución de problemas relacionados con la práctica profesional y calidad de vida por lo cual requiere de la articulación de asignaturas del nivel y disciplina o carrera.

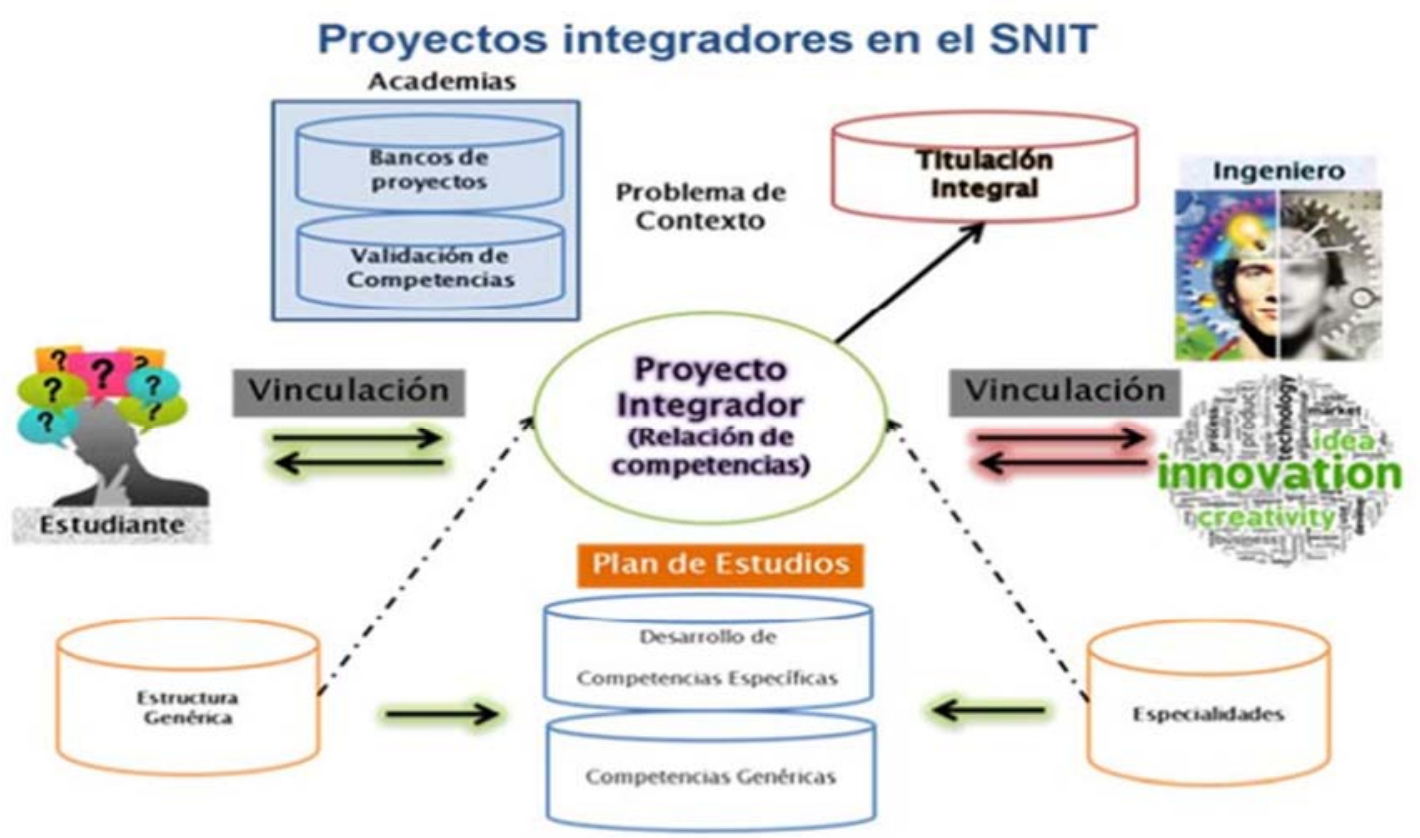

Figura 1. Alcance de los proyectos integradores del Tecnológico Nacional de México.

Fuente: Tecnológico Nacional de México. 
PROYECTOS INTEGRADORES. UNA ESTRATEGIA PARA DESARROLLAR PROYECTOS SUSTENTABLES EN EL AULA

Para la implementación de un proyecto integrador en un currículo diseñado por asignaturas basta sólo seguir tres pasos:

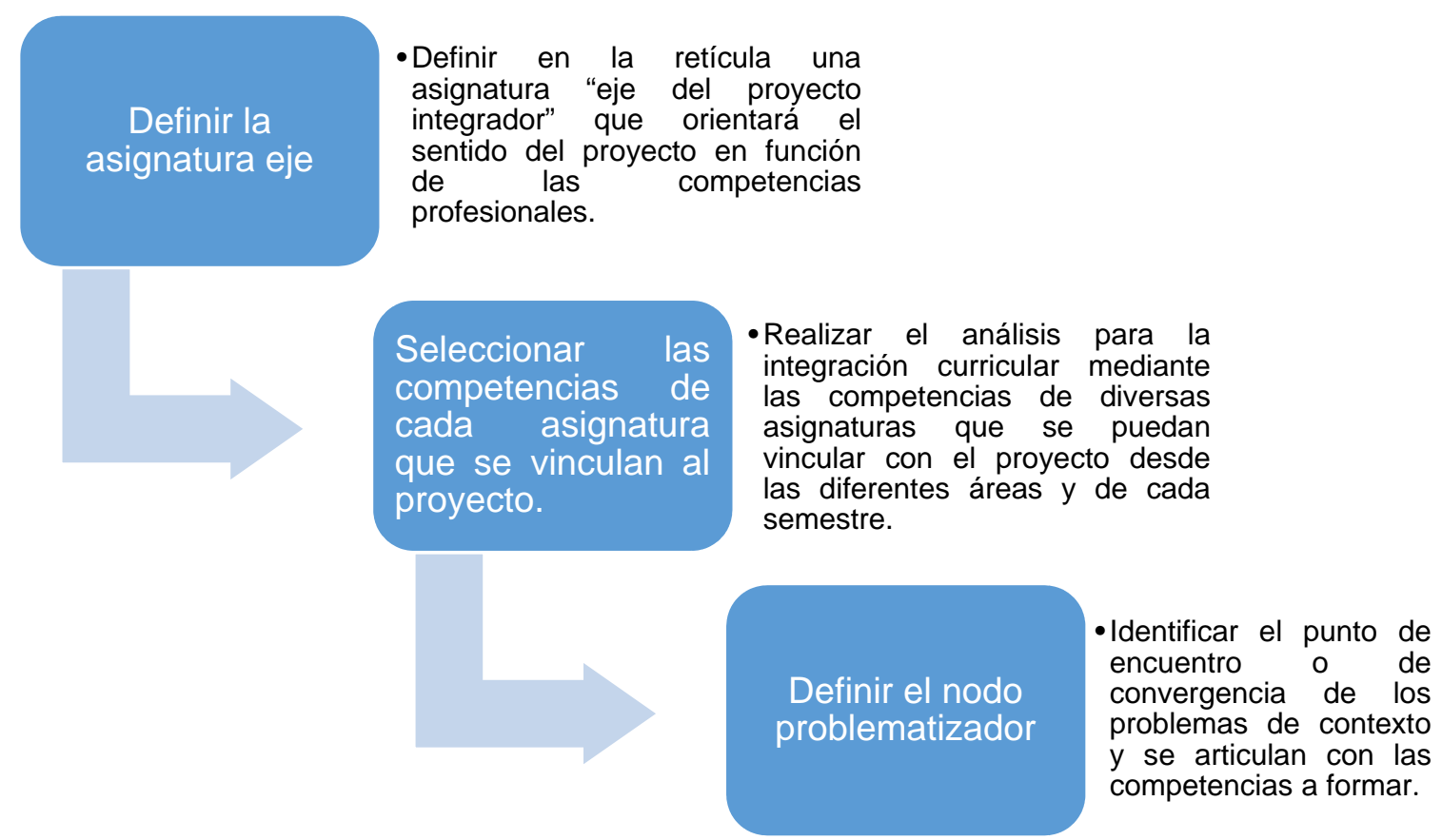

Figura 2. Procesos de los proyectos integradores.

Fuente: Diseño propio basado en, proyectos integradores para la información y desarrollo de competencias profesionales del Tecnológico Nacional de México.

\section{Clases de proyectos integradores}

Como estrategia curricular, los proyectos integradores se pueden implementar en una gran diversidad de proyectos, pero se pueden generalizar en función de cuatro aspectos centrales de un diseño curricular con enfoque en competencias:

- De acuerdo con las competencias que se enfatizan:

o Proyecto genérico: Cuando el proyecto que se va a realizar, se plantea en un conjunto de acciones que direccione el desarrollo de las competencias genéricas establecidas en un plan de estudios.

o Proyecto específico: Cuando el direccionamiento es enfatizado a las competencias específicas de dicho plan. 
PROYECTOS INTEGRADORES. UNA ESTRATEGIA PARA DESARROLLAR PROYECTOS SUSTENTABLES EN EL AULA

o Proyecto global: Cuando se direccionan de manera integral las competencias genéricas y específicas del plan de estudios.

- Por la relación que se establece con las disciplinas:

o Proyecto disciplinario: Si el proyecto se integra por un conjunto de asignaturas de un mismo plan de estudios,

o Proyecto interdisciplinario: Cuando el proyecto integre un conjunto de asignaturas de diversos planes de estudio.

- Por su enfoque:

o Proyecto de investigación: Si en el desarrollo del proyecto, se orienta al estudiante en busca de que aprenda a organizar, sistematizar y analizar la información de un hecho para obtener conclusiones que puedan enriquecer el campo de conocimiento.

o Proyecto tecnológico: Si en el desarrollo del proyecto, se orienta al estudiante en busca del diseño, construcción y transferencia de tecnología (equipos, instrumentos, procesos, entre otros).

o Proyecto social: Cuando el estudiante intenta identificar, diagnosticar y resolver problemas sociales vinculados con la comunidad y su entorno.

o Proyecto económico: Orienta al estudiante a emprender proyectos para generar autoempleos y economías.

o Proyecto cultural: Se desarrolla para crear o recrear mediante estrategias recreativas, deportivas y artísticas.

- Por el grado de participación de los estudiantes.

o Nivel operativo: Si en esta participación el estudiante solo se limita a ejecutar el proyecto sin la realización de sugerencias, mejoras o trabajo colaborativo. 
PROYECTOS INTEGRADORES. UNA ESTRATEGIA PARA DESARROLLAR PROYECTOS SUSTENTABLES EN EL AULA

o Nivel intermedio: Cuando propone la estructuración y construcción de las acciones a realizar durante la ejecución del proyecto.

o Nivel estratégico: Si el estudiante es capaz de proponer un proyecto en conjunto con el profesor o de modificar uno ya existente, (López Rodríguez, 2012).

\section{MATERIAL Y MÉTODO}

En la carrera de Contador Público que se imparte en el Instituto Tecnológico de Cuautla desde hace cuatro generaciones se ha utilizado como estrategia didáctica el Proyecto Integrador "Desarrollo Empresarial", como un mecanismo que permite integrar los conocimientos, habilidades y destrezas adquiridas de las diversas asignaturas del plan de estudio en un proyecto de creación de sus propias empresas, como fuente de autoempleo y considerando las necesidades sociales, económicas y sobre todo cuidando el medio ambiente.

De acuerdo a la clasificación anteriormente mencionado se trabaja con un proyecto: global, disciplinario (aunque no es limitativo), con un enfoque de investigación con base en los aspectos sociales, ecológicos y económicos; y en el cual todos los participantes asumen un rol estratégico.

Objetivo: Conjuntar y relacionar los programas de las diversas materias de la retícula de Contador Público con la finalidad de obtener un aprendizaje significativo y mejores resultados en proyectos que se consoliden o trasciendan en el sector productivo.

Metodología: Cada una de las materias del Plan de estudios aporta a la realización del Proyecto, por lo que desde el primer semestre se hará énfasis en los temas que de alguna manera aportan aspectos al Proyecto final.

En 3er. y $4^{\circ}$. Semestre se da inicio de manera formal el proyecto con las materias de Mercadotecnia, Desarrollo Sustentable y Taller de Investigación I, donde los alumnos se integrarán por equipos con su propuesta de producto y/o servicio teniendo presente la necesidad e importancia de una cultura sustentable.

En $6^{\circ}$. Semestre se incorpora la asignatura Formación de Emprendedores cuyo propósito es desarrollar el interés en el emprendedurismo como un cambio en los paradigmas de los recién 
PROYECTOS INTEGRADORES. UNA ESTRATEGIA PARA DESARROLLAR PROYECTOS SUSTENTABLES EN EL AULA

egresados de las instituciones de educación superior preocupados por tocar puertas en la búsqueda de oportunidades de trabajo sin considerar que dichas oportunidades están en sus manos.

El docente encargado de cada asignatura proporcionará los conocimientos necesarios para la realización del trabajo y evaluará lo correspondiente a la misma. En el $7^{\circ}$. Semestre tres asignaturas trabajaran de forma más intensa para la conclusión del proyecto, cada una aportando con los temas y competencias respectivos: Formulación y evaluación de Proyectos de Inversión, que proporciona la estructura formal al documento, lo que la convierte en materia eje. Las asignaturas de Seminario de Contaduría y Administración Estratégica complementan el proyecto con diversos temas. Al finalizar el curso se llevará a cabo la evaluación mediante presentación en stand y defensa del proyecto, con apoyo de invitados externos expertos de diversos sectores que apoyan la evaluación. Durante el desarrollo de los proyectos los alumnos integrantes de los equipos muestran sus avances en diversos foros como eventos de emprendedores, ferias profesiográficas en escuelas de nivel medio superior y/o congresos, así como en las presentaciones finales de proyectos que se realizan en la institución.

Una vez concluido el proyecto, en la asignatura Técnicas de negociación y comercialización Internacional ( $8^{\circ}$. Semestre), se plantearán las oportunidades de exportación del producto o servicio generado.

\section{Beneficios:}

- Facilita el aprendizaje del estudiante, generando conocimientos integrados

- Resuelve problemas del contexto incorporando el saber, el saber ser y el saber hacer en las actividades del proyecto.

- Desarrolla en el estudiante capacidades y destrezas cognitivas, afectivas, sociales y de resolución de problemas.

- Evalúa la pertinencia y actualidad de los programas educativos.

- Incrementa la productividad tecnológica y el registro de patentes y marcas.

- Propicia el trabajo colaborativo entre docentes y alumnos. 
PROYECTOS INTEGRADORES. UNA ESTRATEGIA PARA DESARROLLAR PROYECTOS SUSTENTABLES EN EL AULA

\section{RESULTADOS}

Lógicamente no es realista pensar que todos los proyectos generados en el aula deben convertirse en empresas, pero, si se puede generar la inquietud emprendedora en algunos de los egresados se estará cumpliendo con el objetivo.

Actualmente, algunos de estos proyectos se encuentran en la etapa de incubación dentro del Centro de Innovación e Incubación Empresarial del Instituto Tecnológico de Cuautla con empresas de diversos giros. Otros más han realizado sus Residencias Profesionales u obtenido su Título Profesional con sus proyectos. Algunos de ellos han participado en el Evento Nacional Estudiantil de Emprendedores del TecNM y año con año se pretende incrementar la participación de alumnos con sus proyectos en diversos foros.

Independientemente de todos los beneficios anteriormente citados, los proyectos integradores familiarizan a los estudiantes con un panorama completo e interrelacionado de las organizaciones y no con temas aislados, lo sitúan en una posición más realista de lo que será su desempeño profesional. Apoyan y obligan a trabajar en equipo, no solo a los alumnos, ya que el tipo de actividades se extiende también a los docentes.

\section{CONCLUSIONES}

Día con día en las IES se fomenta cada vez más las actividades interdisciplinarias, por lo que los Proyectos integradores nos proporcionan la oportunidad no solo de incentivar el trabajo en equipo, sino de hacerlo multidisciplinario tratando con esto de impulsar la creatividad e innovación de los estudiantes.

Es importante también que los alumnos participen en foros, congresos u otras actividades, en la cuales puedan dar a conocer sus proyectos, tratando de que estos no sean solo un trabajo de aula, sino que se conviertan en empresas que impulsen el desarrollo económico de la región. 
PROYECTOS INTEGRADORES. UNA ESTRATEGIA PARA DESARROLLAR PROYECTOS SUSTENTABLES EN EL AULA

\section{REFERENCIAS BIBLIOGRÁFICAS}

Dirección General de Educación Superior Tecnológica. (2009). Guía para la instrumentación didáctica de los programas de estudio para la formación y desarrollo de competencias profesionales. México: DGEST.

Dirección General de Educación Superior Tecnológica. (2009). Metodología para el diseño e innovación curricular para la formación y desarrollo de competencias profesionales. México: DGEST.

Dirección General de Educación Superior Tecnológica. (2012). Modelo educativo para el siglo XXI: Formación y desarrollo de competencias profesionales. México: DGEST.

Molina, Ruíz, E. (2007). La Práctica Profesional, componente de formación en la preparación de futuros profesionales. Investigación Educativa, 11(19), 19-34. Recuperado de http://go.galegroup.com.ezproxylocal.library.nova.edu/ps/retrieve.

Paz, H. (2007). El aprendizaje situado como una alternativa en la formación de competencias en ingeniería. Colombia: Revista Educación en Ingeniería, 2(4). Recuperado de http://go.galegroup.com.ezproxylocal.library.nova.edu/ps/retrieve.

Tamayo y Tamayo, M. (1994). Metodología formal de la investigación científica. México: Limusa.

Tamayo y Tamayo, M. (1999). Módulo 5: El proyecto de investigación. Serie Aprender a investigar. México: ICFES. Recuperado de: https://es.scribd.com/document/212893762/5El-Proyecto-de-Investigacion-APRENDER-A-INVESTIGAR-ICFES.

Tamayo y Tamayo, M. (2003). El proceso de la investigación científica. México: Limusa.

Tecnológico Nacional de México. (2012) Proyectos integradores para la formación y desarrollo de competencias profesionales del Tecnológico Nacional de México. México. TecNM.

Tobón, S. (2010). Formación integral y competencias. Pensamiento complejo, currículo, didáctica y evaluación. Bogotá: Ecoe. 
PROYECTOS INTEGRADORES. UNA ESTRATEGIA PARA DESARROLLAR PROYECTOS SUSTENTABLES EN EL AULA

Tobón, S. P. (2010). Secuencias didácticas: aprendizaje y evaluación de competencias. México: Pearson.

Tobón, S. (2010). El modelo de competencias en las prácticas docentes: Hacia escenarios significativos de vida. México: Conrrumbo.

Van Dalen, D. B., \& Meyer, W. J. (1974). Manual de técnicas de investigación educacional. Buenos Aires: Paidós.

Whitney, F. L. (1970). Elementos de investigación. Barcelona: Alfa Omega. 